\title{
Multi-Wavelength Laser Transmitter for the Two-Step Laser Time-of-Flight Mass Spectrometer
}

Anthony W. Yu, Steven X. Li, Molly E. Fahey, Andrej Grubisic, Benjamin J. Farcy, Kyle Uckert, Xiang Li and Stephanie Getty NASA Goddard Space Flight Center

Greenbelt, MD

CLEO 2017

15 MAY 2017 
- Motivation

- L2MS Instrument Overview

- L2MS Laser Architecture

- Preliminary Performance

- Future Work \& Conclusions 
- Motivation

- L2MS Instrument Overview

- L2MS Laser Architecture

- Preliminary Performance

- Future Work \& Conclusions 


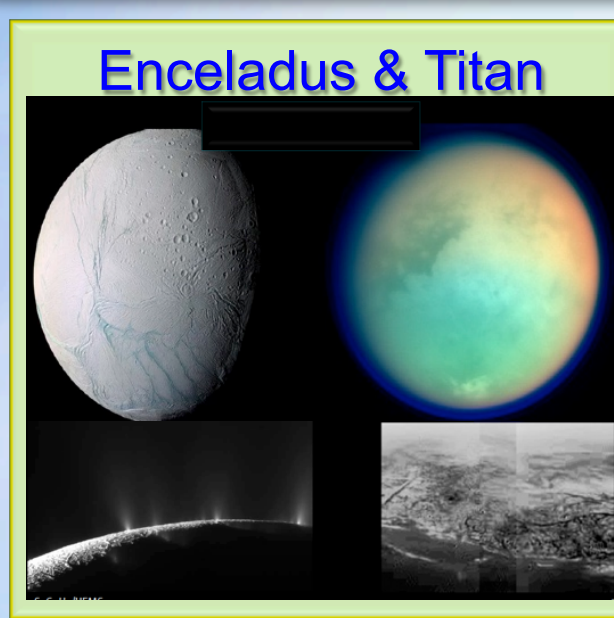

\section{Europa}
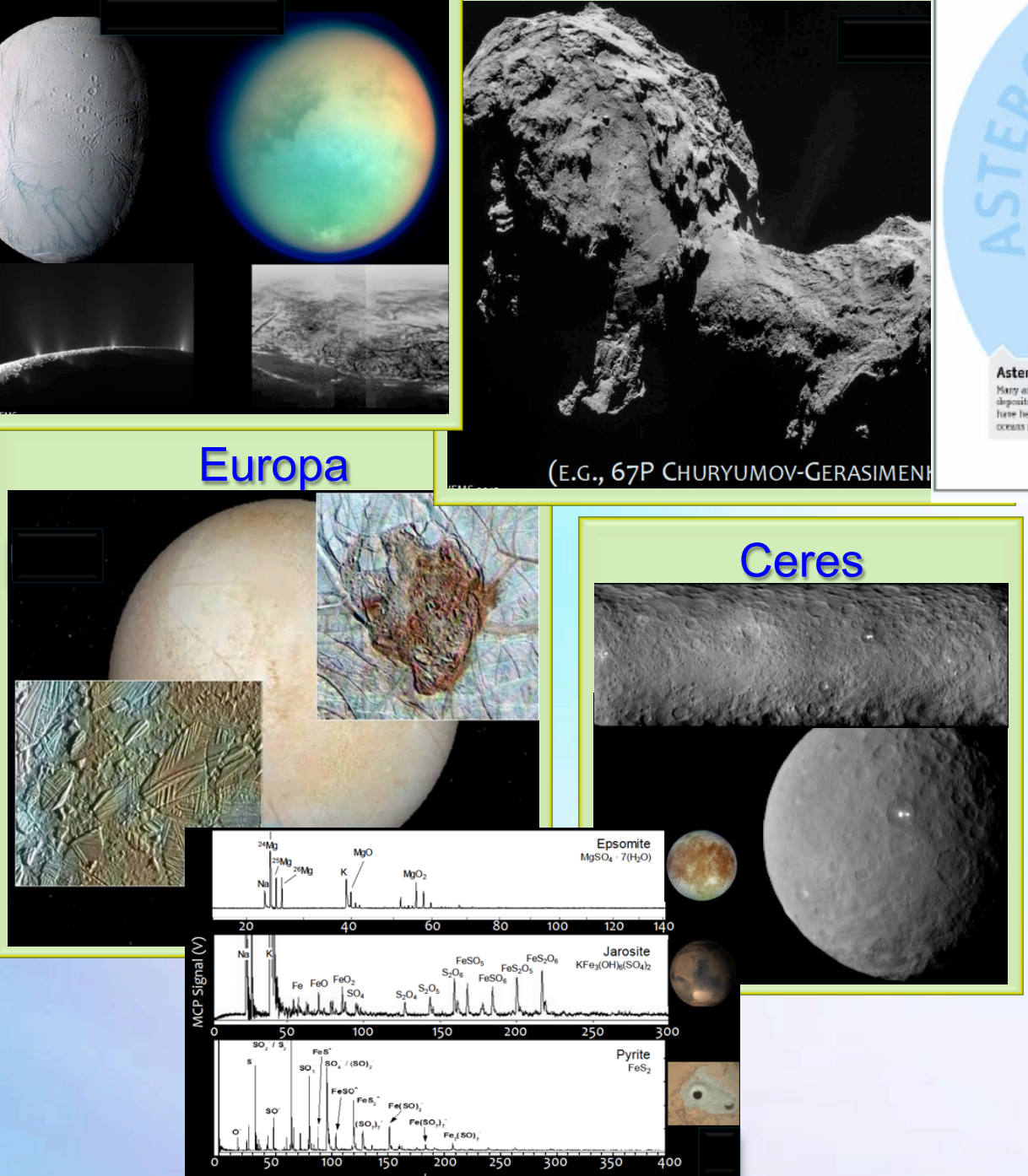

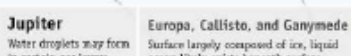

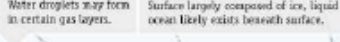

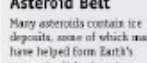

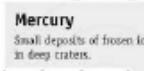

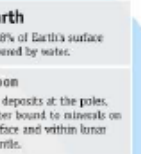

.

SOL 90

○

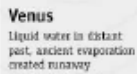

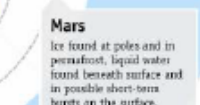

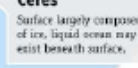

\section{All these bodies contain volatiles, including} water just waiting to be analyzed

Our science motivation is to seek a solution with "Universal" detector for

- Comprehensive sample analysis

- Flexibility to adapt for different mission architectures including flybys, orbiters, landers, and/or rovers! 
- Motivation

- L2MS Instrument Overview

- L2MS Laser Architecture

- Preliminary Performance

- Future Work \& Conclusions 


\section{L2MS Instrument Overview}

\section{Laser Desorption/Ionization Mass Spectrometry}

UV Ionization Pulse

Sample

MIR Desorption Puls

\section{Neutral}

Plume

Time-of-flight Mass

Spectrometer 
- Wavelengths are selected based on key vibrational and electronic resonances in the targeted species aligned with the organic diversity and mineralogy expected for future planetary missions of high priority to NASA

- $2.75 \mu \mathrm{m}-\mathrm{IR}$ vibrational resonances of hydrated minerals

- $\quad 3.4 \mu \mathrm{m}-\mathrm{C}-\mathrm{H}$ vibration resonance of organic species

- $266 \mathrm{~nm}$ - coincides with a short-lived metastable state in many aromatic molecules

- Matching MIR laser wavelength allows for selective desorption
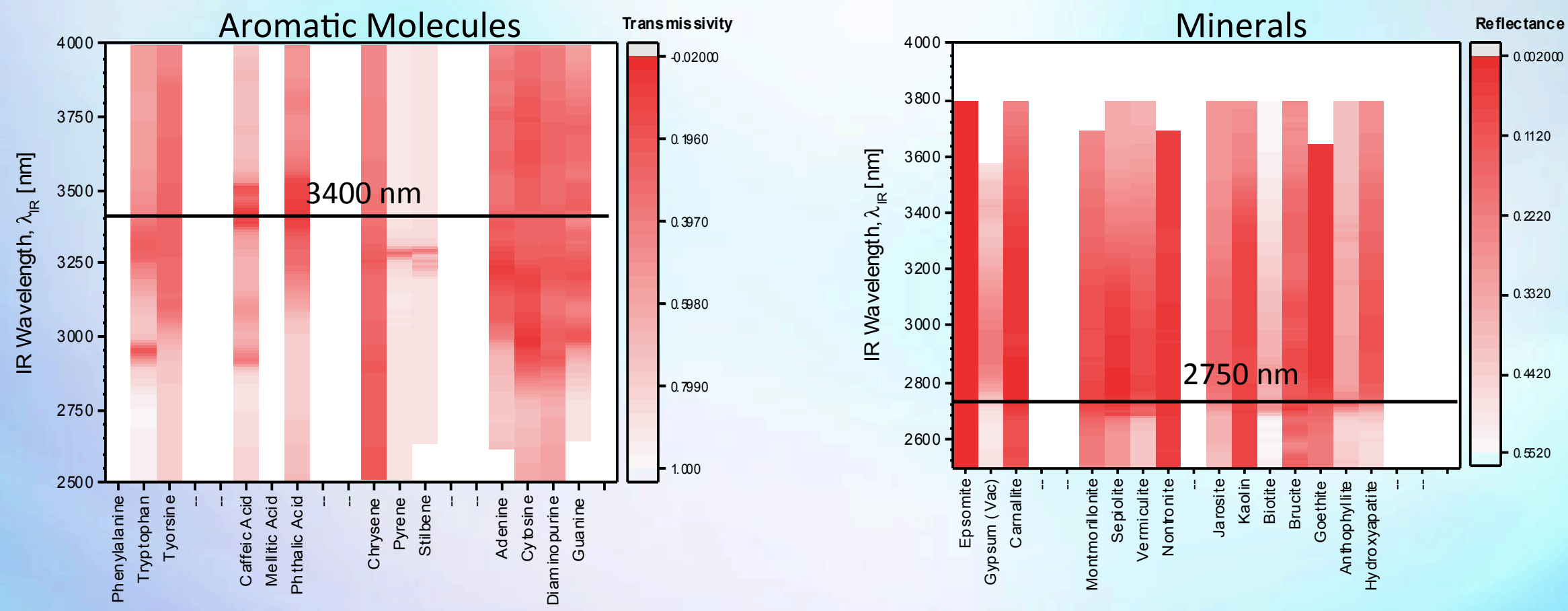


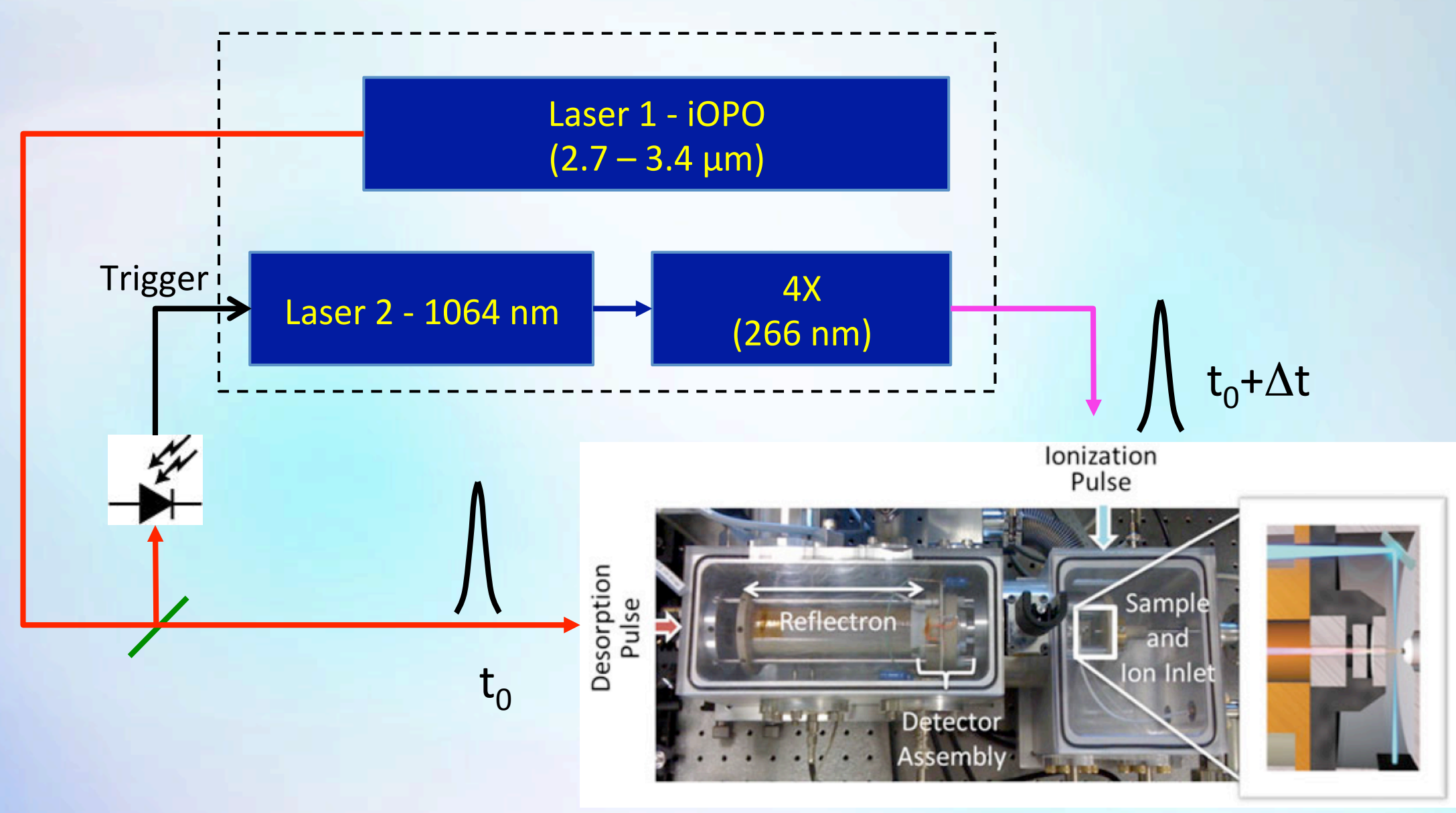

- Typical delay between Laser 1 and Laser $2(\Delta \mathrm{t})$ range between 0.3-2 $\mu \mathrm{s}$ 
- Motivation

- L2MS Instrument Overview

- L2MS Laser Architecture

- Preliminary Performance

- Future Work \& Conclusions 


\begin{tabular}{|c|c|c|}
\hline Lasers Requirement & MIR Laser & UV Laser \\
\hline Pulse Repetition Frequency (PRF) & $1-20 \mathrm{~Hz}$ & $1-20 \mathrm{~Hz}$ \\
\hline Wavelength & $2.8 \mathrm{X} \mu \mathrm{m}$ and $3.40 \pm 0.05 \mu \mathrm{m}$ & $266 \mathrm{~nm}$ \\
\hline Energy & $\sim 100 \mu \mathrm{J}$ & $\sim 18 \mu \mathrm{J}$ \\
\hline Pulse Width & $<7 \mathrm{~ns}$ & $<7 \mathrm{~ns}$ \\
\hline Peak Power & $\sim 14 \mathrm{~kW}$ & $\sim 2.5 \mathrm{~kW}$ \\
\hline Peak Intensity (assuming $100 \mu \mathrm{m}$ beam diameter) & $180 \mathrm{MW} / \mathrm{cm}^{2}$ & $\sim 30 \mathrm{MW} / \mathrm{cm}^{2}$ \\
\hline Spectral Width & Few GHz & Few $\mathrm{GHz}$ \\
\hline Timing & $t_{0}$ & $\mathrm{t}_{\mathrm{o}}+\Delta \mathrm{t} ; \sim 100 \mathrm{~ns}<\Delta \mathrm{t}<\mathrm{few} \mu \mathrm{s}$ \\
\hline Laser Lifetime & \multicolumn{2}{|c|}{3 year mission at $10 \%$ duty cycle 64 Mshots @ $20 \mathrm{~Hz}$} \\
\hline
\end{tabular}


- Laser design for both Laser 1 (MIR) and Laser 2 (UV) is based on the previously flown Lunar Orbiter Laser Altimeter (LOLA) laser transmitter

\begin{tabular}{|c|c|}
\hline Instrument & LOLA \\
\hline Mission & LRO \\
\hline Launched & $6 / 18 / 2009$ \\
\hline Laser type & Cr:Nd:YAG, 5 way beam split \\
\hline Laser Architecture & $\begin{array}{c}\text { Cross-Porro resonator passively } \\
\text { Q-switched }\end{array}$ \\
\hline \# of lasers & 2 \\
\hline Laser Wavelength & $1064.3 \pm 0.1 \mathrm{~nm}$ \\
\hline Laser pulse energy & $2.7 \pm 0.3 \mathrm{~mJ}$ \\
\hline $\begin{array}{c}\text { Laser Pulse Repetition } \\
\text { Rate }\end{array}$ & $28.0 \pm 0.1 \mathrm{~Hz}$ \\
\hline Laser Pulsewidth & $6.0 \pm 2.0 \mathrm{~ns}$ \\
\hline Laser Beam quality & TEM00 \\
\hline Laser Divergence & $100 \mu \mathrm{rad}(=5 \mathrm{~m}$ footprint) \\
\hline
\end{tabular}

As of 3/2016, the number of shots fired by each lasers are: LOLA laser transmitter

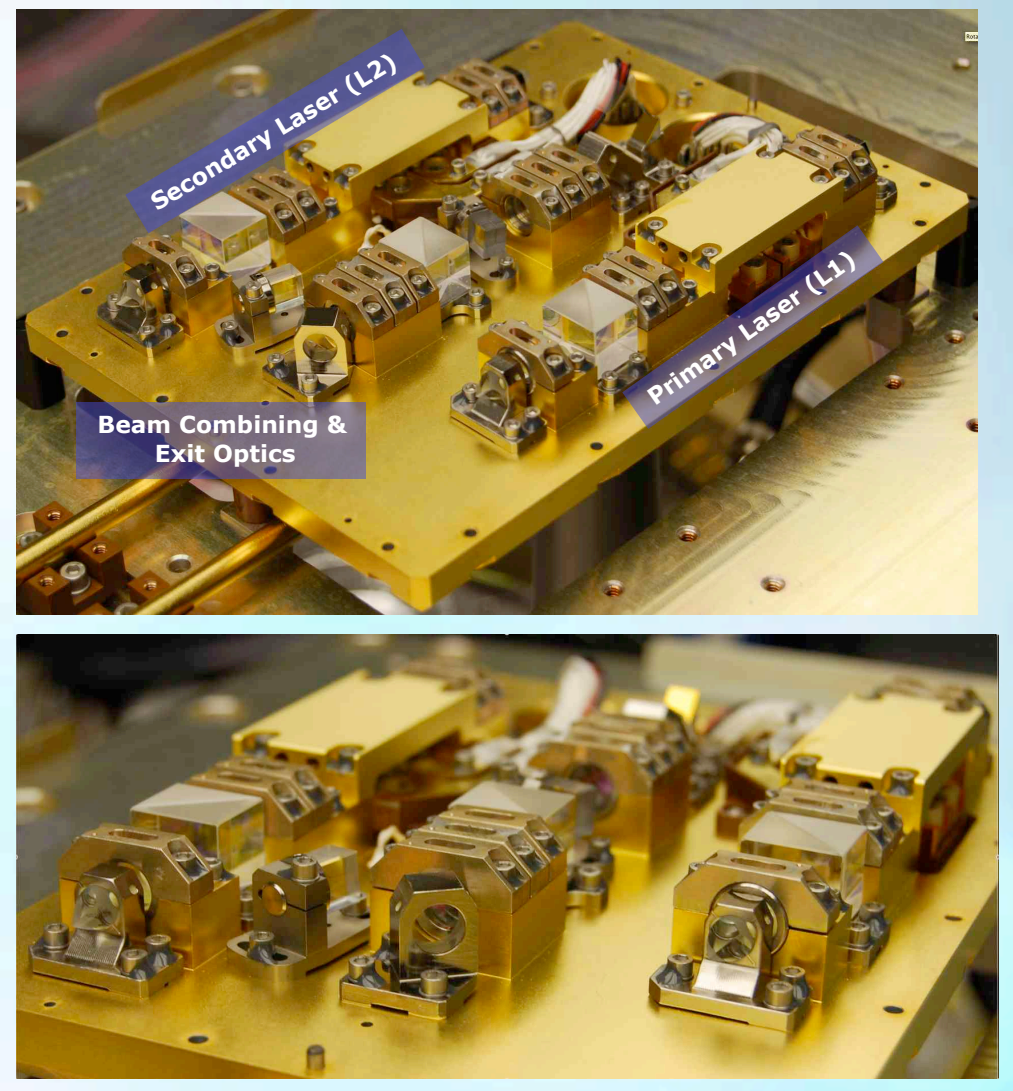

- $\quad$ Laser $1 \sim 2.1 \mathrm{~B}$

- Laser $2 \sim 2 \mathrm{~B}$ 


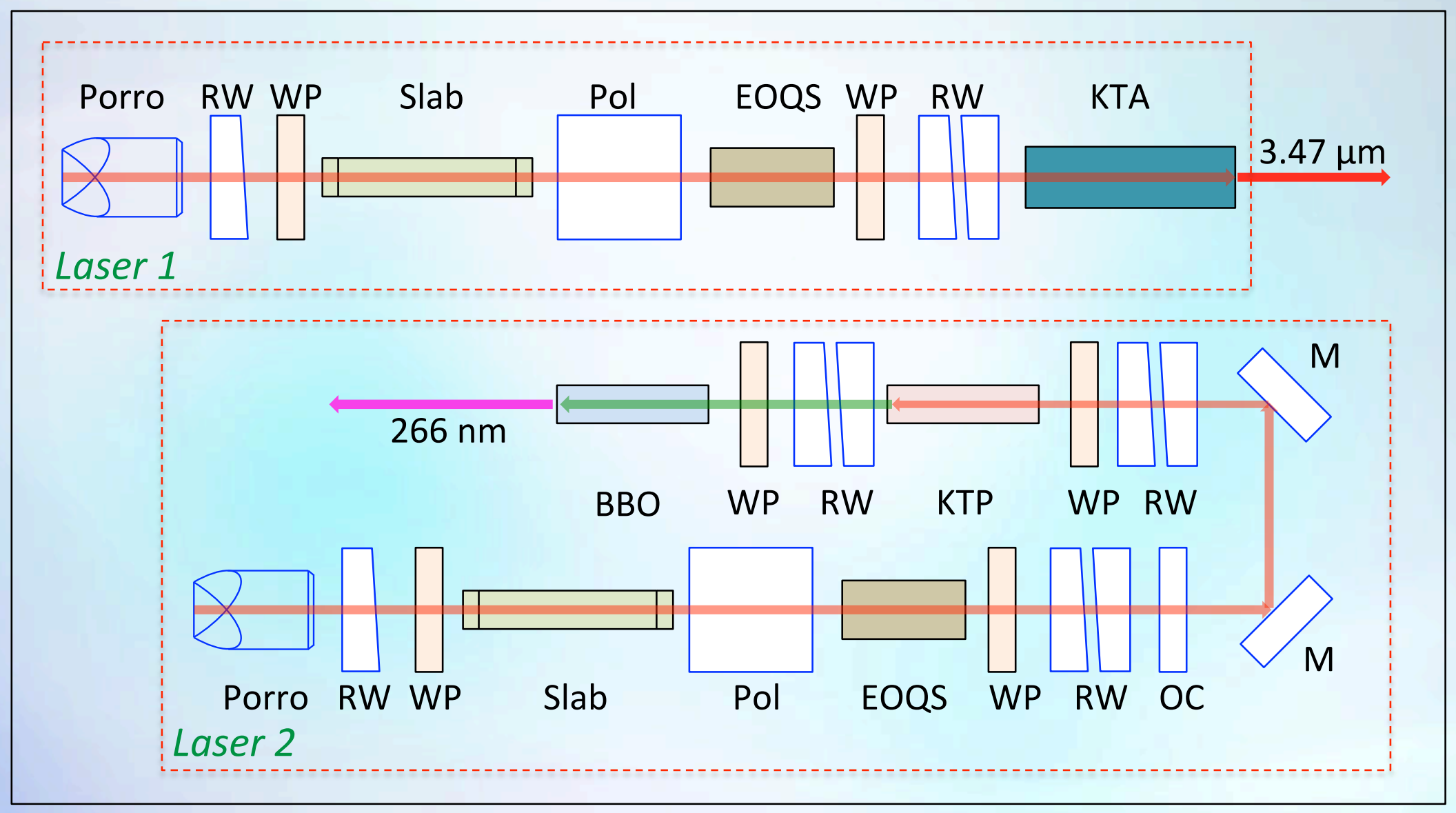

PP - Porro Prism; RW - Risley Wedge; WP - Wave plate; Pol -

Polarizer; EOQS - Pockels Cell; M - Mirror; OC - Output Coupler 


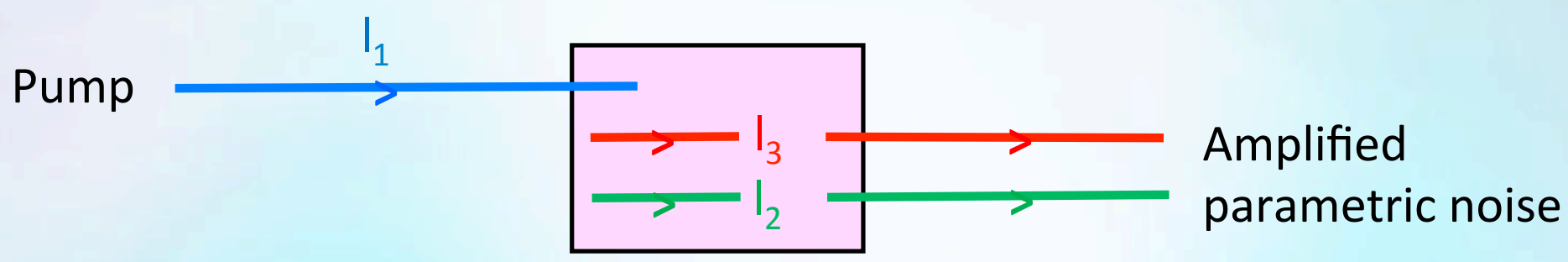

Input pump, hc/ $\mathrm{I}_{1}$, spontaneously generates pairs of photons, $\mathrm{hc} / \mathrm{I}_{2}, \mathrm{hc} / \mathrm{I}_{3}$ (parametric noise) which are then amplified.

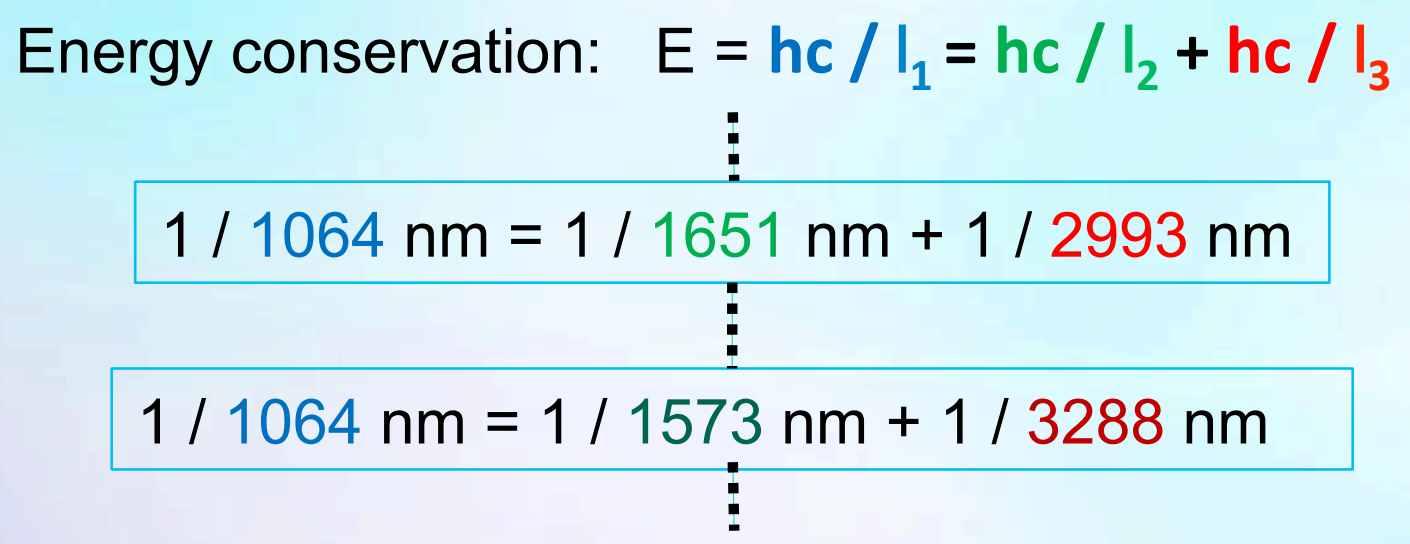




\section{Optical parametric oscillation}

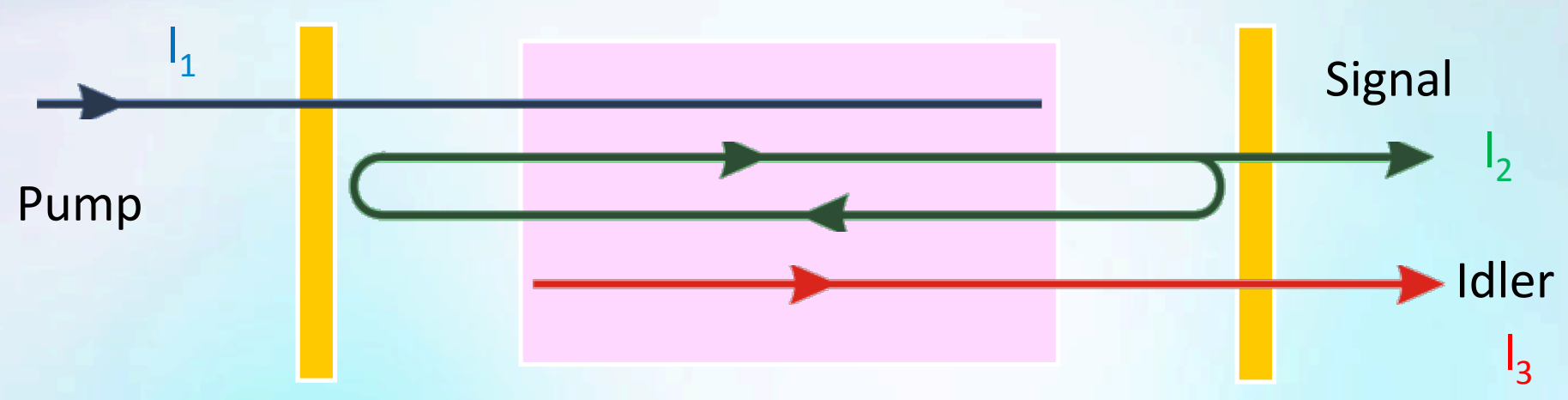

Singly-resonant oscillator (SRO)

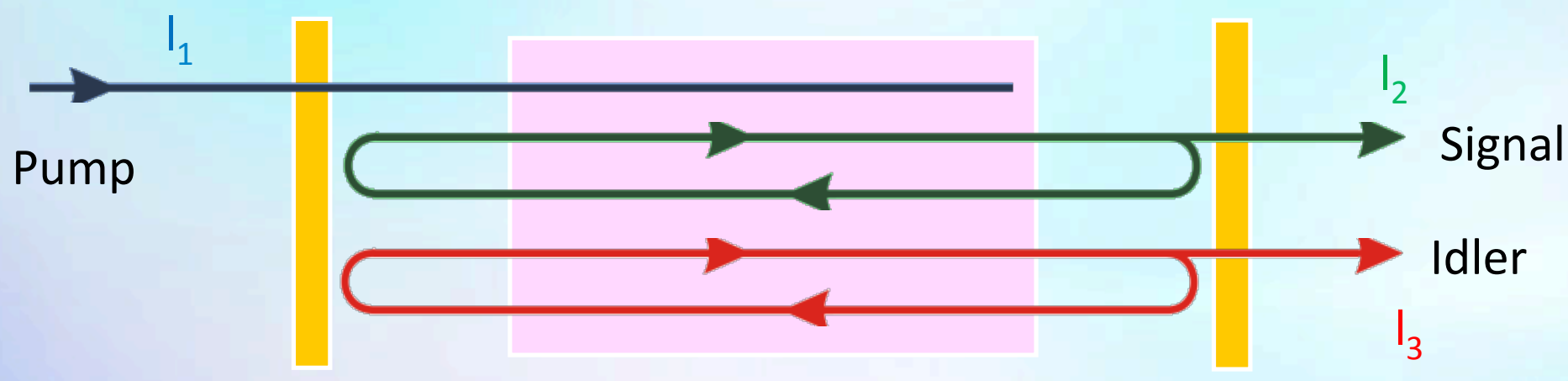

Doubly-resonant oscillator (DRO) 


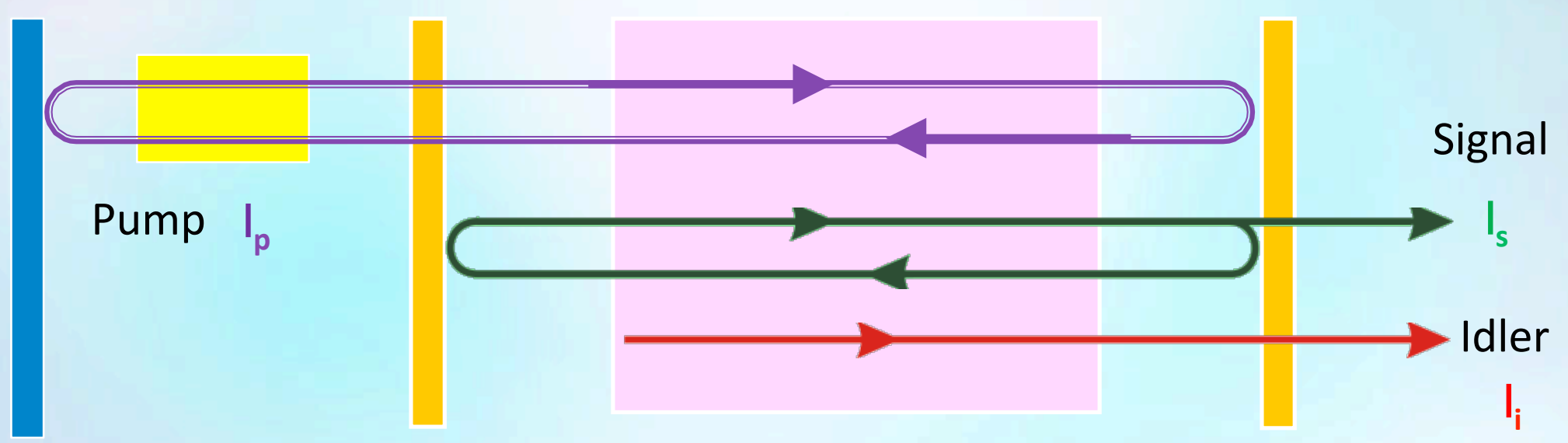

$M_{1} \quad M_{2}$ 


\section{Nonritical phase matching (NCPM)}

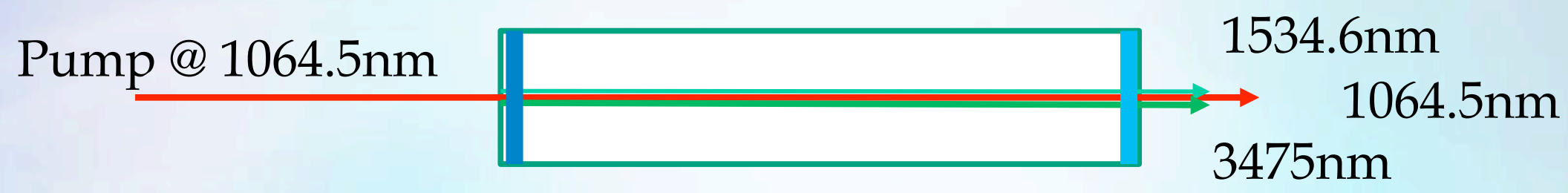

Critical phase matching

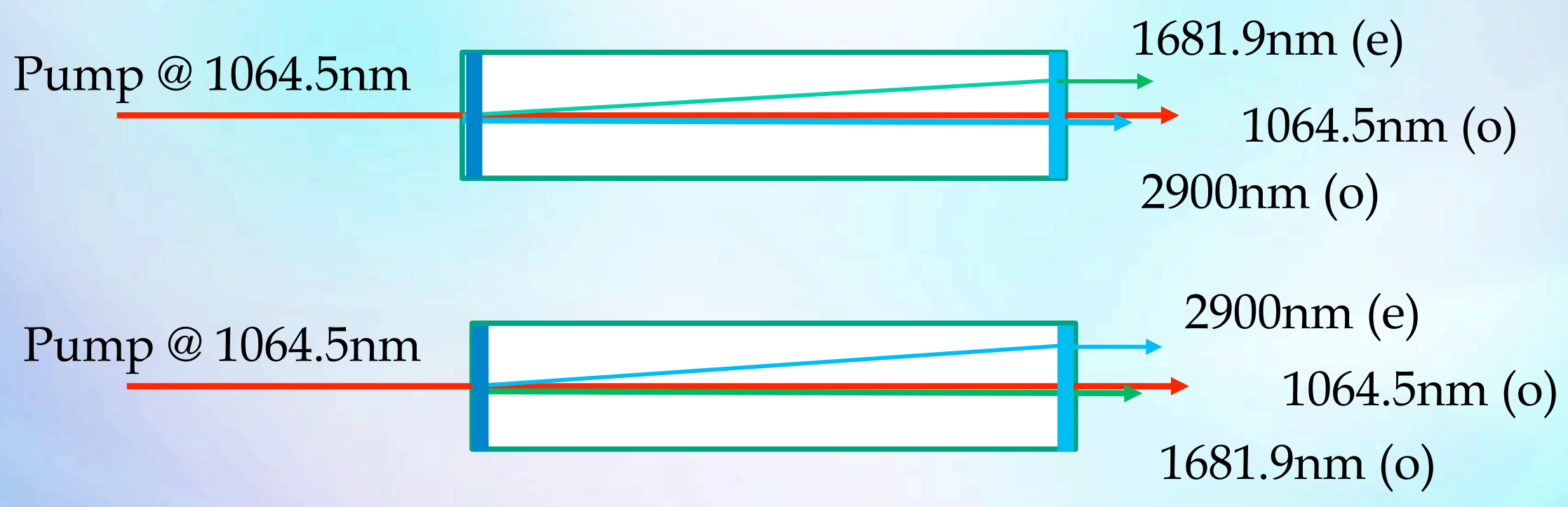




\section{Monolithic iOPO Design}

- Replace LOLA laser output coupling section with a monolithic OPO

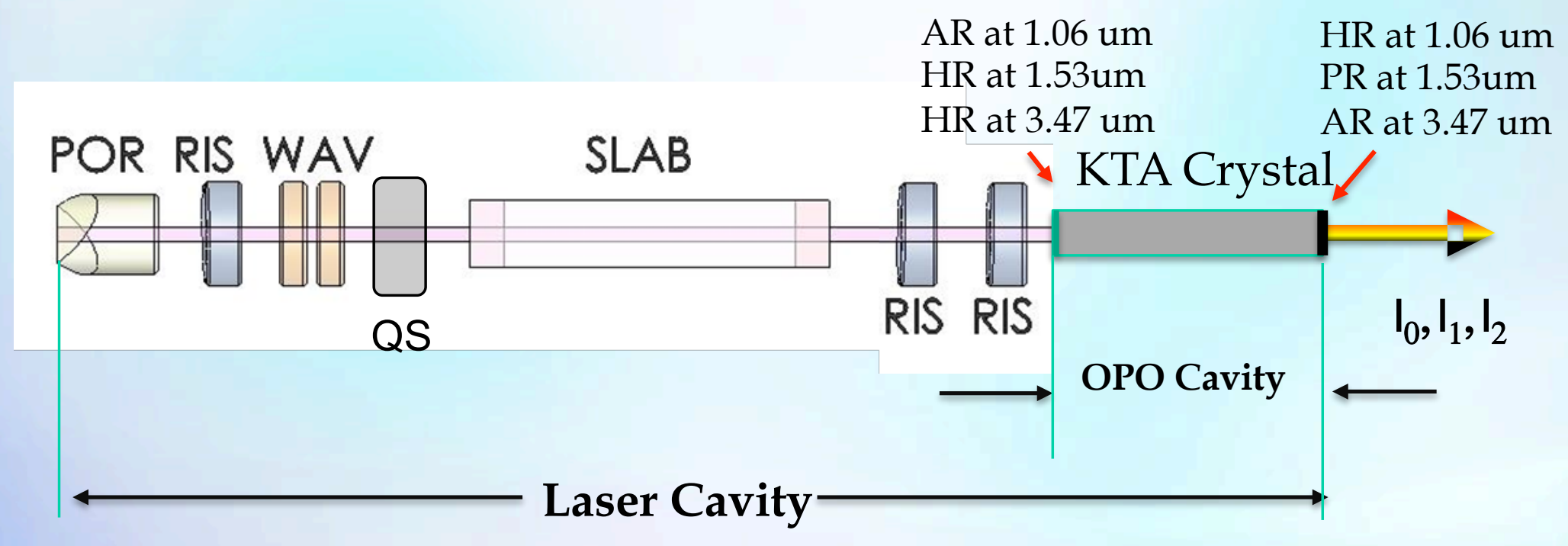




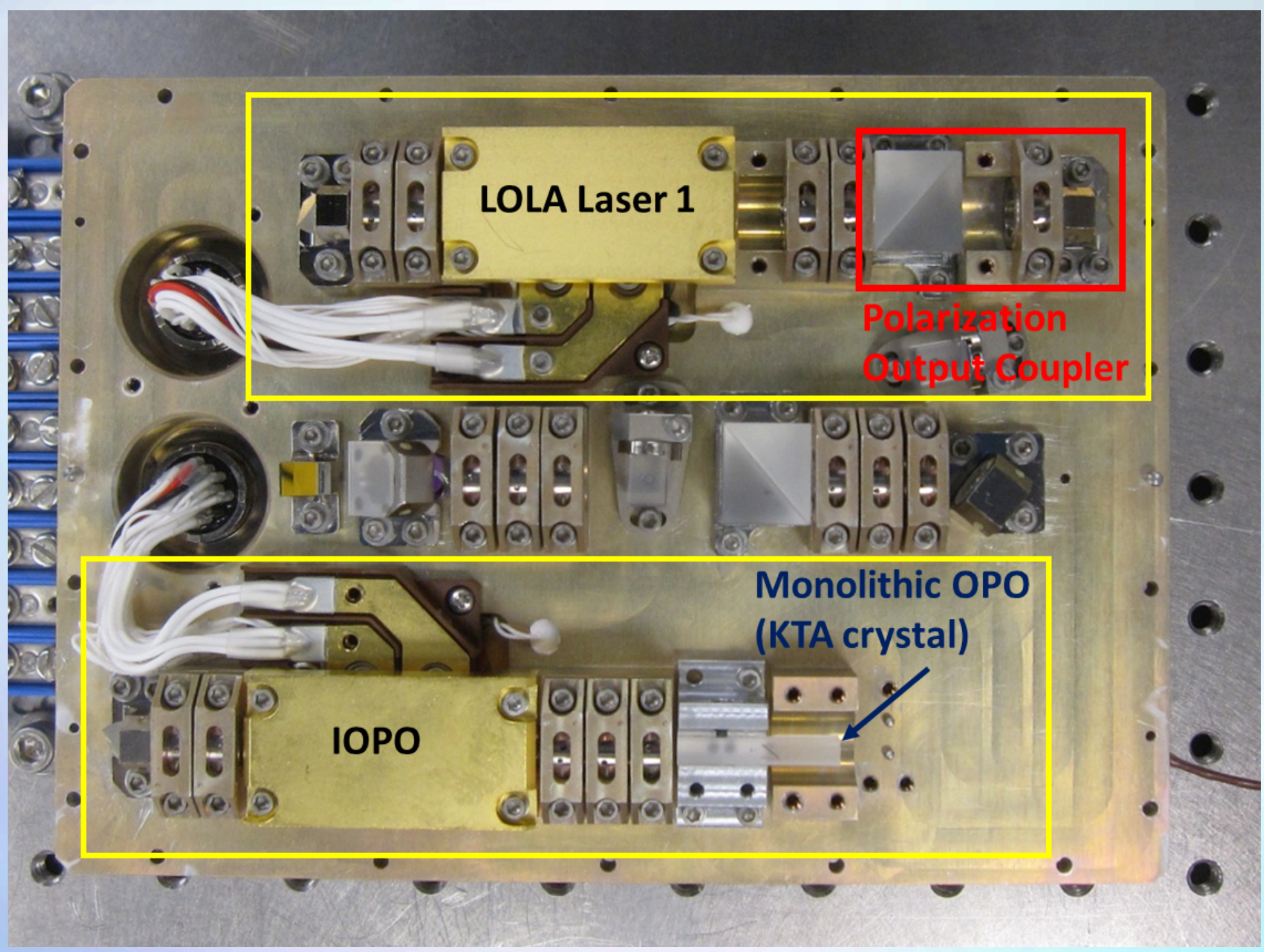


- Motivation

- L2MS Instrument Overview

- L2MS Laser Architecture

- Preliminary Performance

- Future Work \& Conclusions 


\section{iOPO Experimental results, $3.4 \mu \mathrm{m}$}

$$
\begin{aligned}
\text { Output Energy } & =450 \mu \mathrm{J} \text { at } 3471 \mathrm{~nm} \\
& =1.2 \mathrm{~mJ} \text { at } 1534 \mathrm{~nm}
\end{aligned}
$$
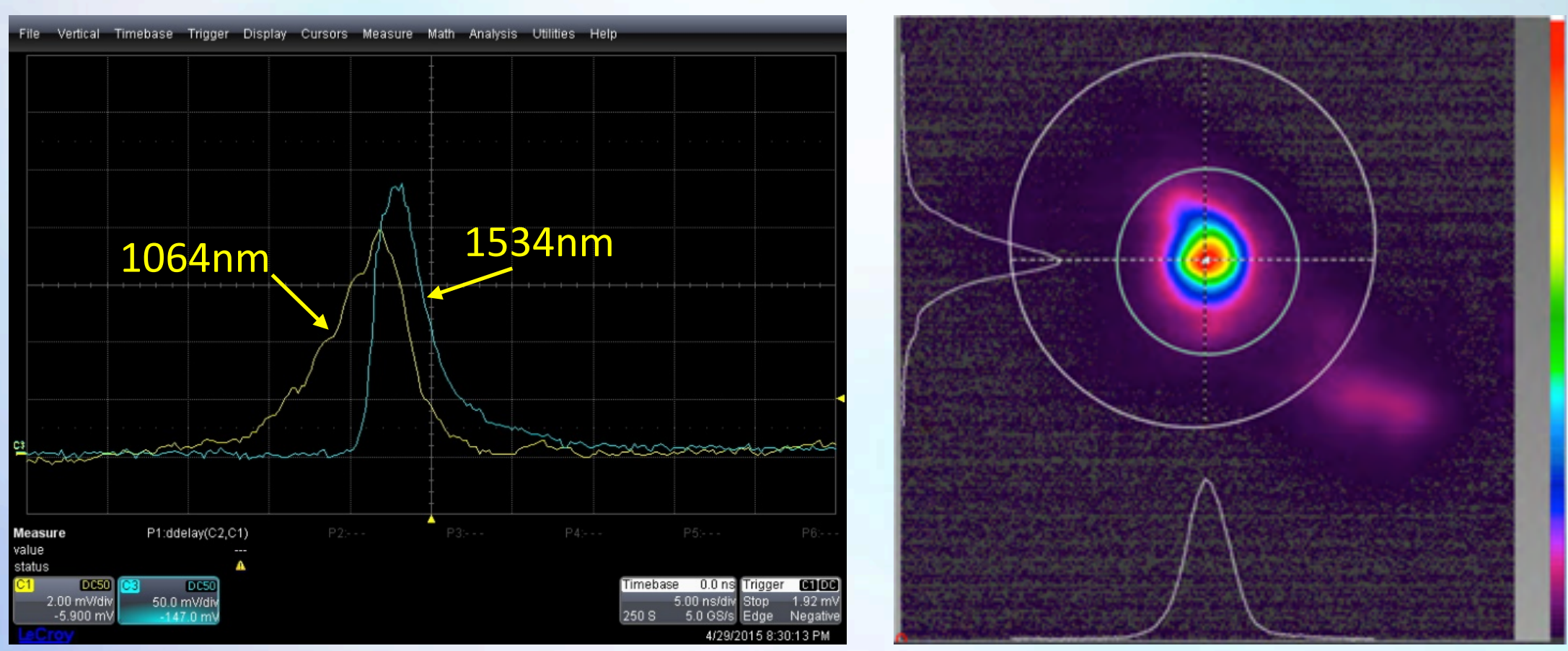


\section{iOPO Experimental result, $3.4 \mu \mathrm{m}$}

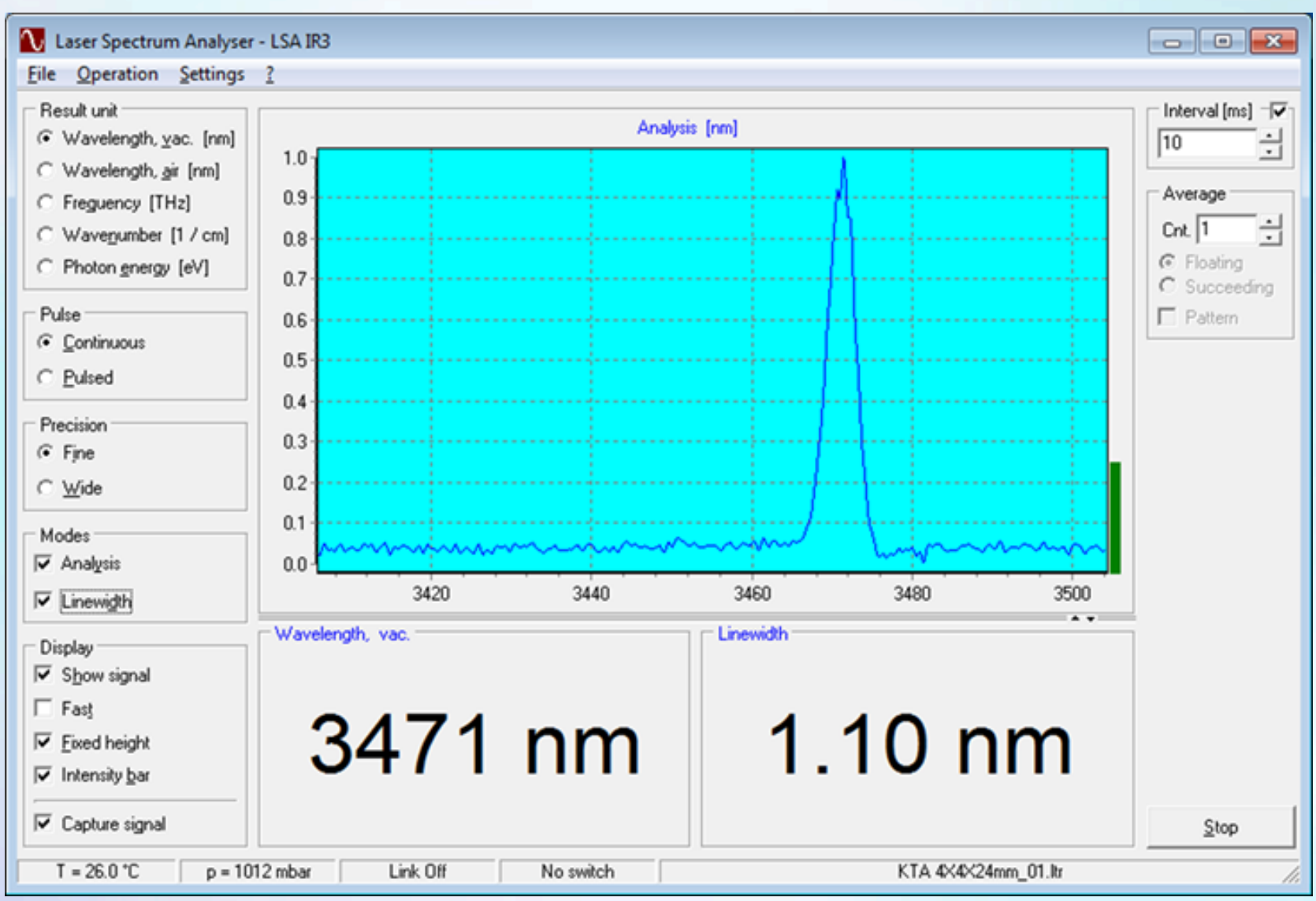




\section{iOPO breadboard using CPM monolithic OPO crystal, $2.7 \& 2.9 \mu \mathrm{m}$}

Critical phase matching
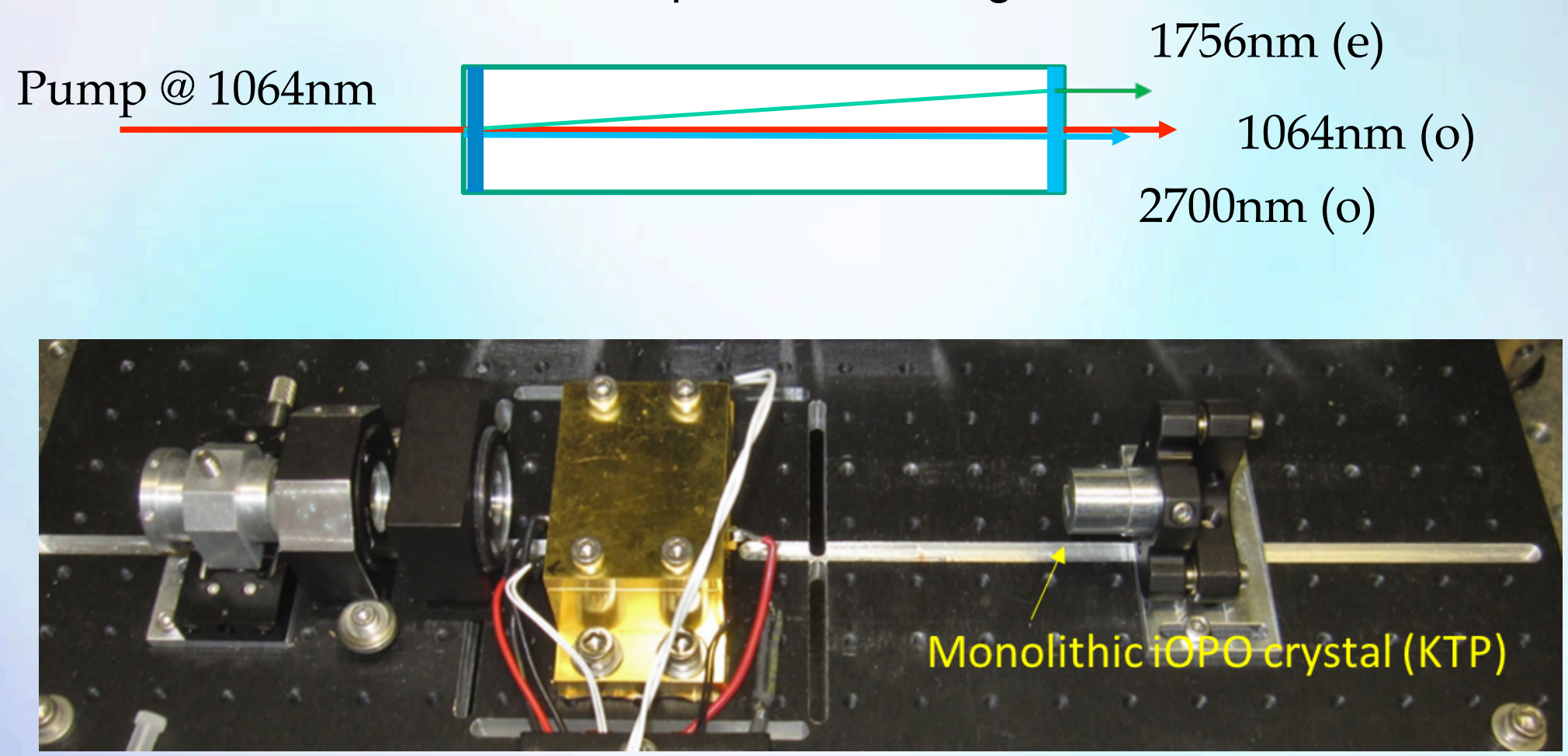


\section{Coating damage near 2.85um}

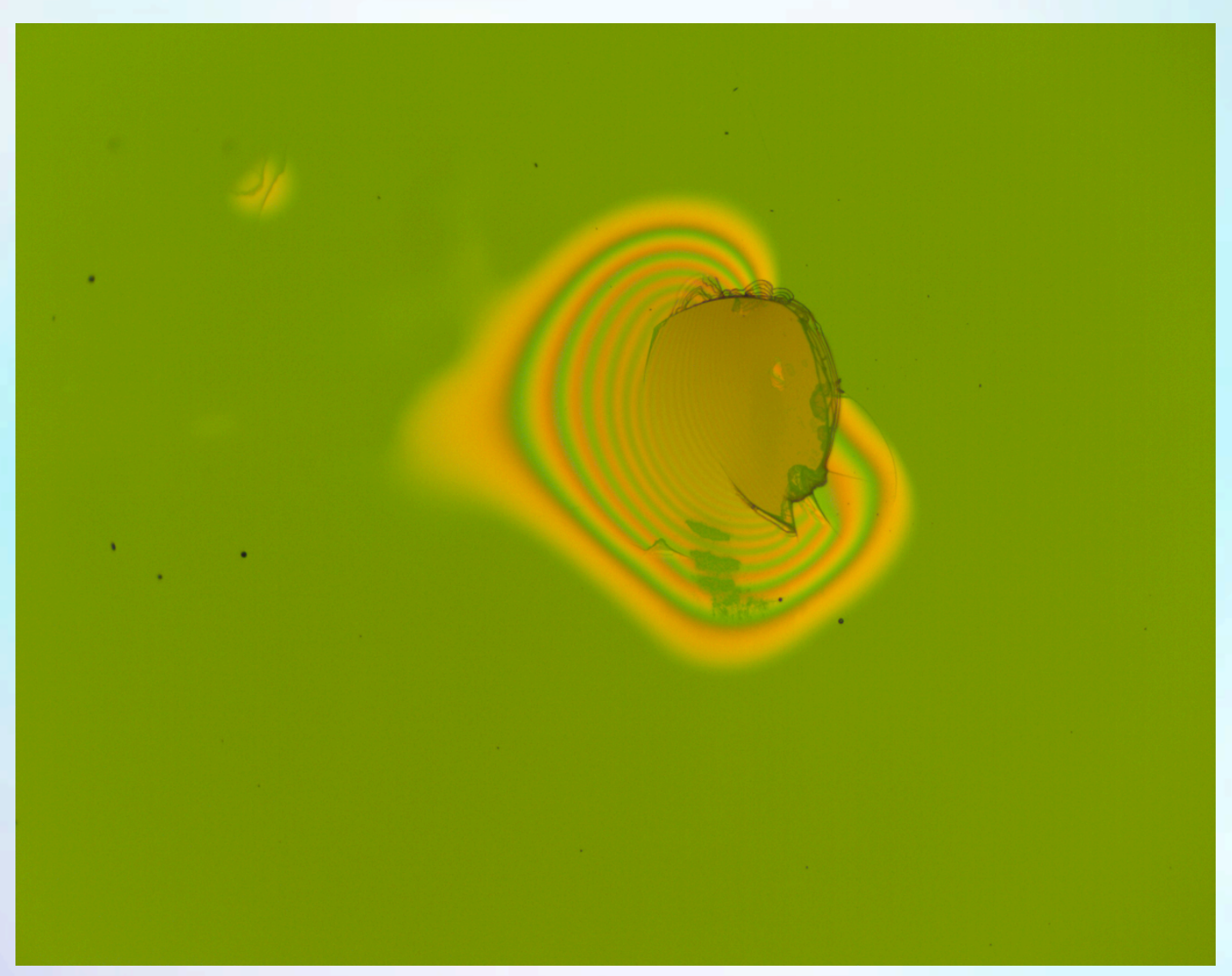



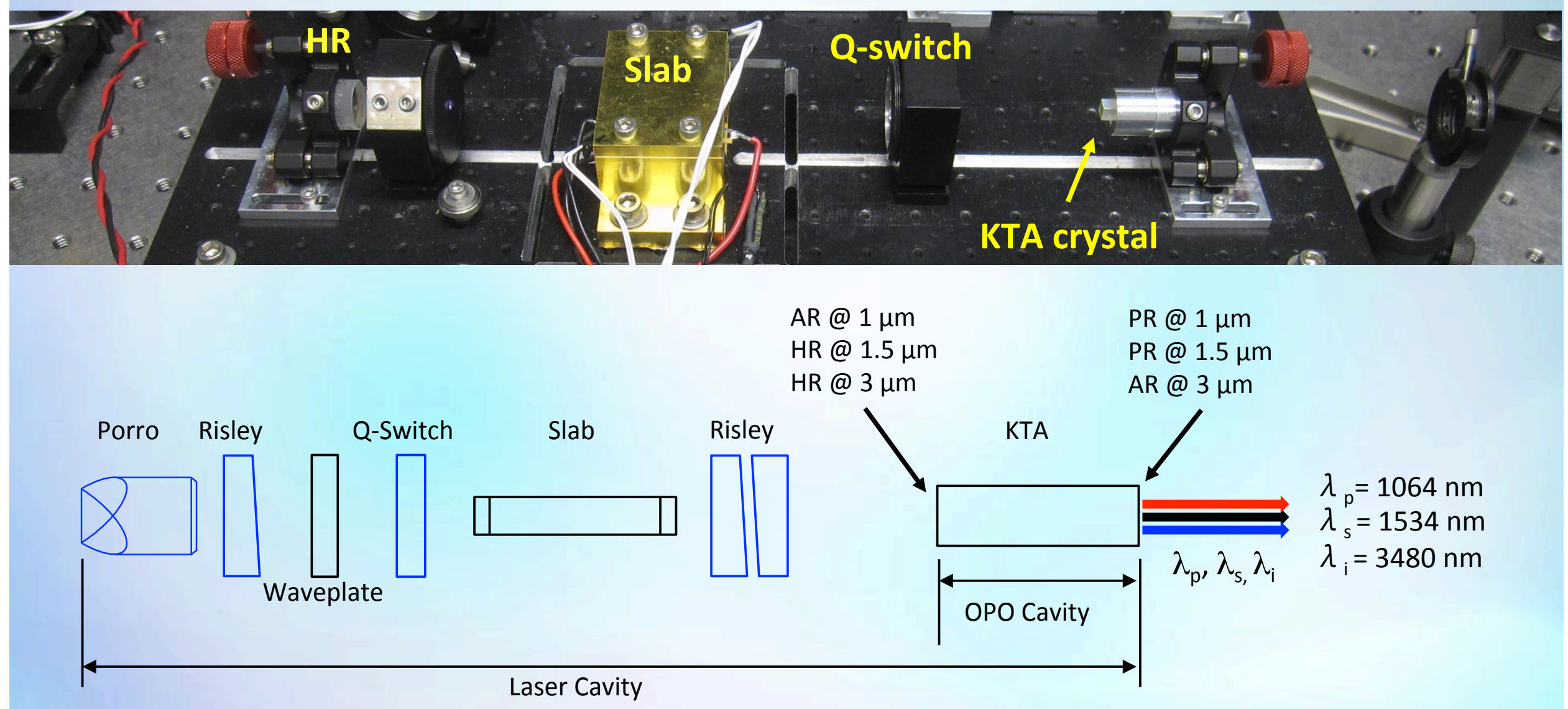


\begin{tabular}{|c|c|c|}
\hline Parameter & Measurement & Requirement \\
\hline Wavelength & $3471 \mathrm{~nm}$ & $3400 \mathrm{~nm} \pm 50 \mathrm{~nm}$ \\
\hline
\end{tabular}

Pulse Repetition Frequency

$20 \mathrm{~Hz}$

$1-20 \mathrm{~Hz}$

(PRF)

Average Power

Energy

$4.2 \mathrm{~mW}$

NA

Energy

$210 \mu \mathrm{J}$

$100 \mu \mathrm{J}$

Conversion Efficiency

$1.9 \mathrm{~ns}$

$<7$ ns

$10.5 \%$

NA

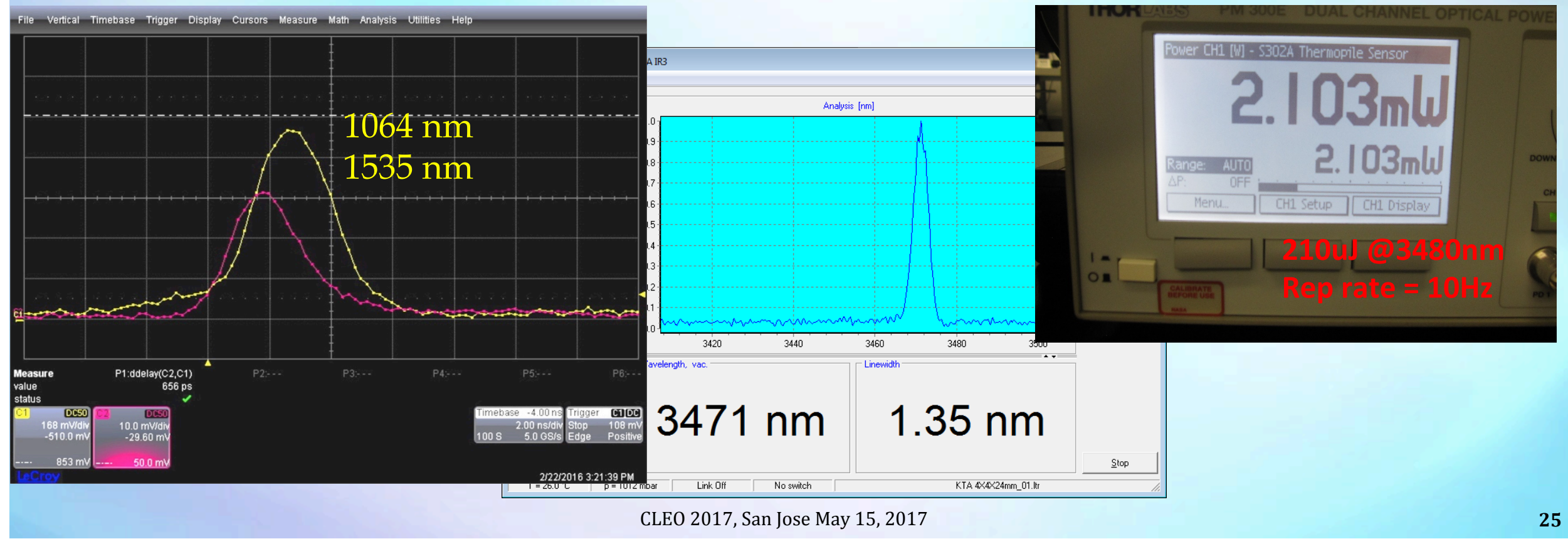




\section{NAST UV Laser Breadboard}

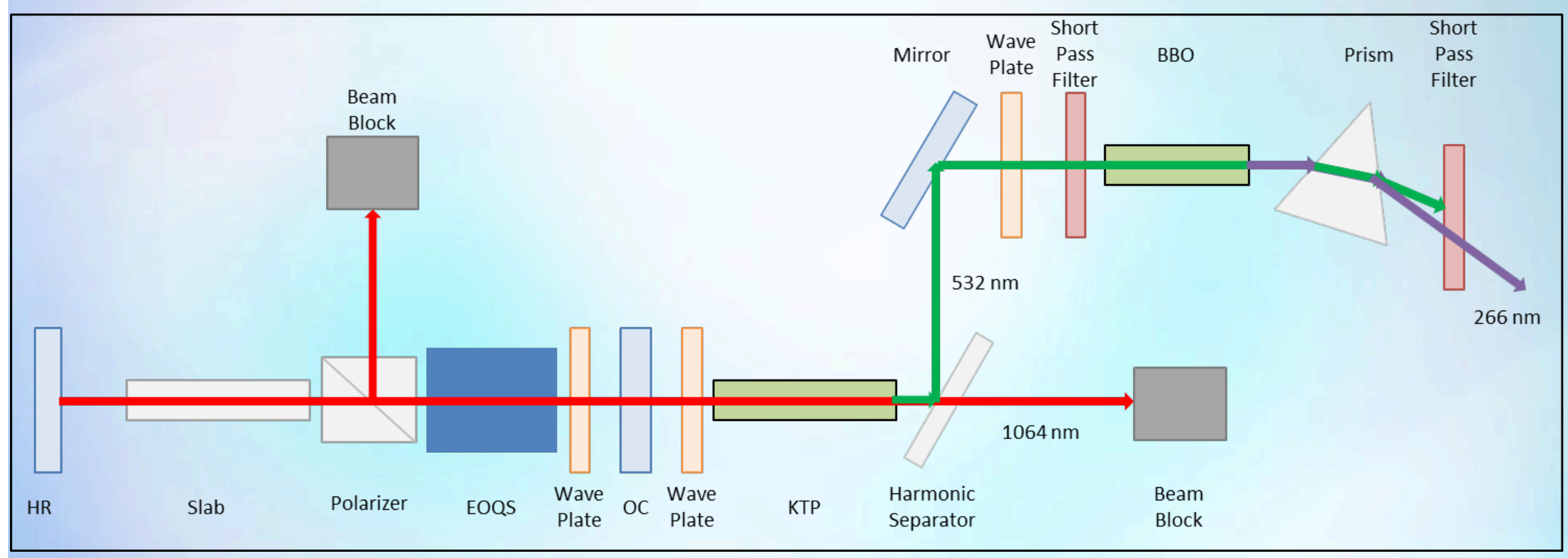




\section{NASA 266 nm Laser Performance}

\begin{tabular}{|c|c|c|}
\hline Parameter & Measurement & Requirement \\
\hline Wavelength & $266.2 \mathrm{~nm}$ & $266 \mathrm{~nm}$ \\
\hline Pulse Repetition Frequency (PRF) & $20 \mathrm{~Hz}$ & $1-20 \mathrm{~Hz}$ \\
\hline Energy & $220 \mu \mathrm{J}$ & $18 \mathrm{~J}$ \\
\hline Pulse Duration & $6.6 \mathrm{~ns}$ & $<7 \mathrm{~ns}$ \\
\hline Peak Power & $32.5 \mathrm{~kW}$ & $2.5 \mathrm{~kW}$ \\
\hline Peak Intensity (assuming $100 \mu \mathrm{mW}$ beam diameter) & $415 \mathrm{MW} / \mathrm{cm}^{2}$ \\
\hline Divergence (full angle) & $\theta_{\mathrm{x}}=0.66 \mathrm{mrad}$ & $\mathrm{NA}$ \\
\hline Overall $^{\text {th }}$ Harmonic Conversion Efficiency & $\theta_{\mathrm{y}}=1.12 \mathrm{mrad}$ & $\mathrm{NA}$ \\
\hline
\end{tabular}
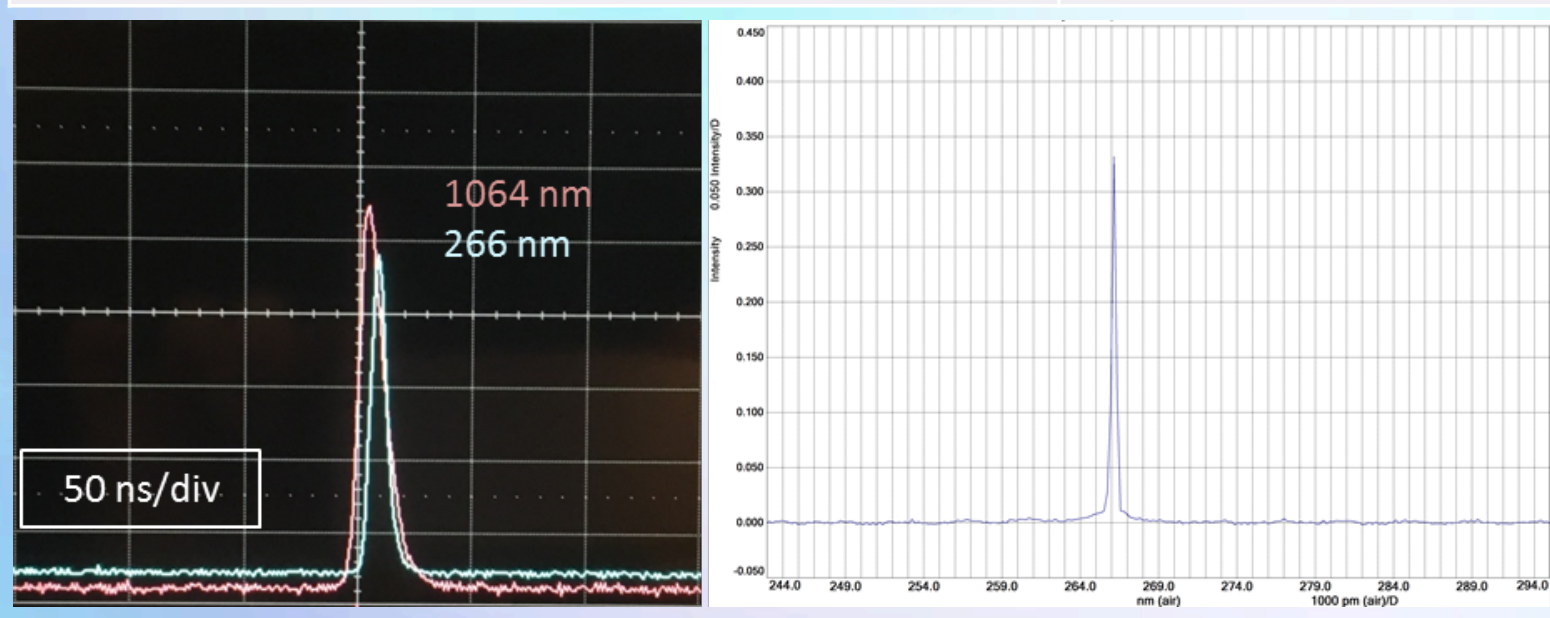

Far Field Beam 


\section{Nas: Integration of Laser to L2MS Instrument}
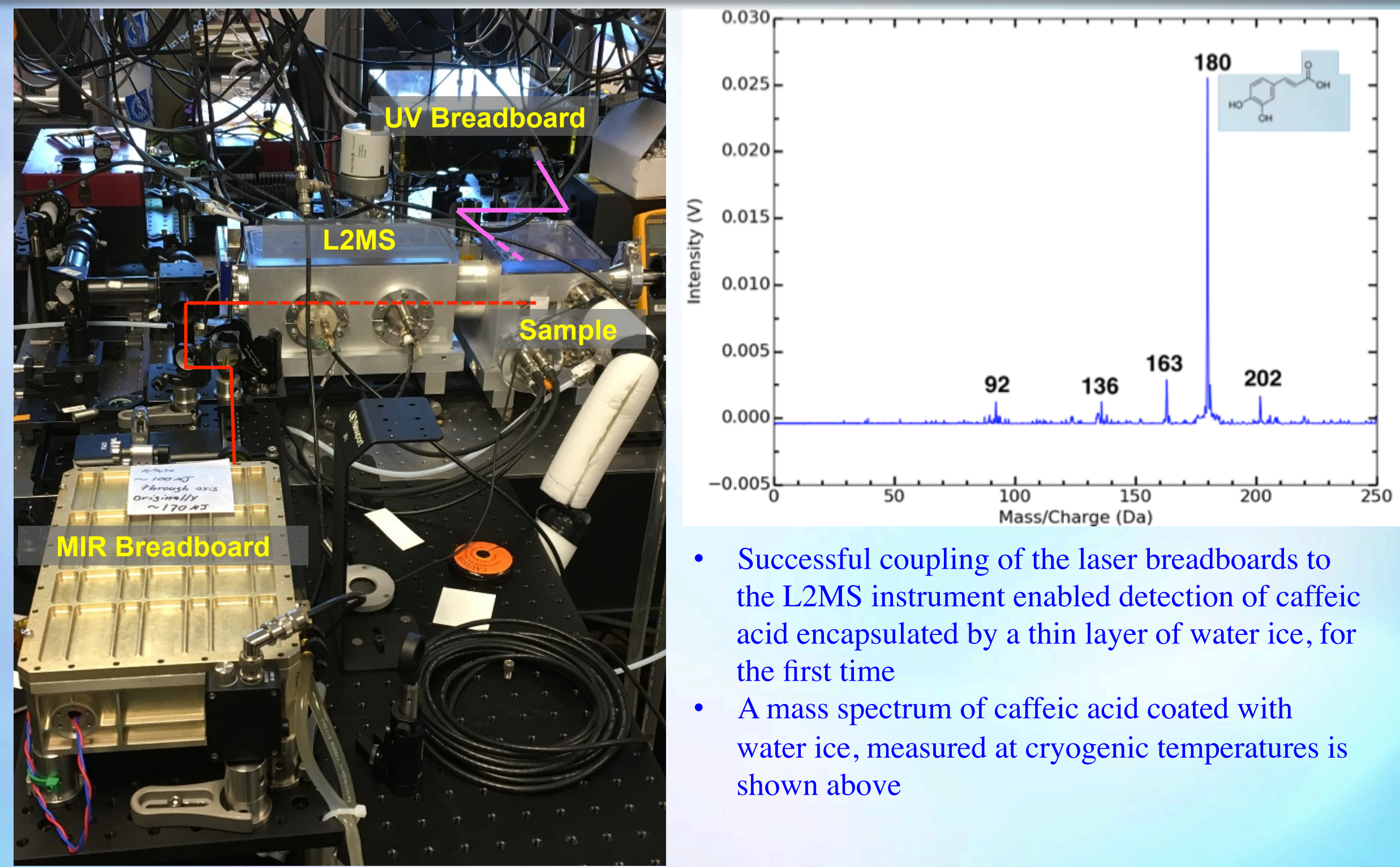

- Successful coupling of the laser breadboards to the L2MS instrument enabled detection of caffeic acid encapsulated by a thin layer of water ice, for the first time

- A mass spectrum of caffeic acid coated with water ice, measured at cryogenic temperatures is shown above 
- Motivation

- L2MS Instrument Overview

- L2MS Laser Architecture

- Preliminary Performance

- Future Work \& Conclusions 


\section{Laser 1 - MIR Laser}

- Test 3.4 $\mu \mathrm{m}$ breadboard with L2MS laboratory instrument and compare with commercial OPO

- Complete $2.75 \mu \mathrm{m}$ breadboard laser

- Finalize design for dual wavelength $(2.75 \mu \mathrm{m}$ and $3.4 \mu \mathrm{m})$ concept

Laser 2 - UV Laser

- Investigate other non-linear optical crystals for SHG and FHG - leverage ICESat-2/ATLAS LBO aging study

- Optimize overall $4^{\text {th }}$ harmonic conversion efficiency

- Test breadboard with L2MS laboratory instrument and compare with commercial UV laser

- Develop epoxy-free opto-mechanical design for mounting optics to minimize UV induced contamination on optical surfaces

\section{Laser Transmitter}

- Improve packaging of the laser transmitter for space flight

- Build brass board laser transmitter that will generate both MIR and UV wavelengths on a single laser bench 
- We are developing a multi-wavelength laser transmitter for the L2MS Instrument

- A new laser architecture based on the LOLA laser transmitter that generates a single discrete MIR and UV wavelengths has been demonstrated

- The approach provides a straightforward path toward space laser design and deployment

- Preliminary laser breadboard results show compliance with the L2MS instrument requirements 